\title{
Permeation Process of Metal Complexes with Hydroxyoxime and Carboxylic Acid Extractants through Organic-Aqueous Interface
}

\author{
Yasutaka NISHIHARA* \\ Sumitomo Metal Mining Co., Ltd.,3-5 Sobiraki-cho, Niihama, Ehime 792-0001, Japan
}

(Received September 30, 2020; Accepted April 22, 2021)

Permeation process of the cobalt(II) (Co) complexes of the hydroxyoxime extractant, LIX63 and/or of the carboxylic acid extractant, Versatic Acid 10 (VA10), through the interface between cyclohexane and water layers was investigated using a hybrid Monte Carlo/Molecular Dynamics (MC/MD) method where MC and MD were performed for coordination reactions probabilistically and molecule movements on short time scale, respectively. For VA10 system, no $\mathrm{Co}^{2+}$ permeated through the interface and this suggests that VA10 are not coordinated directly to $\mathrm{Co}^{2+}$. For Co complexes with LIX63, two LIX63 molecules were coordinated with $\mathrm{Co}^{2+}$ from the organic phase. The complexes rotated and one of the LIX63 molecules was located between $\mathrm{Co}^{2+}$ and the aqueous phase, then the complexes migrated into the organic phase. This suggests that the diffusion of the extractant molecules and the rotation of the complexes around the interface are important for solvent extraction.

\section{Introduction}

In solvent extraction with two immiscible liquids such as organic and aqueous liquids, the liquid-liquid interface influences the kinetics of metal extraction [1-4]. One of the reasons is that water-soluble ligands in metal complexes are replaced by organic extractant molecules at the organic-aqueous interface. In order to improve extraction processes, it is essential to understand the permeation process of metal complexes through the interface.

The extraction efficiency is defined with the ratio of the initial metal concentration in the aqueous phase and the extracted metal concentration in the organic phase, that is, the energy difference between the metal complexes in the aqueous and organic phases. An increase in extractant efficiency is possible by usage of multiple extractants known as synergistic extraction [5,6] and development of novel extractant molecules [7]. In novel extractant development, molecules are designed to stabilize metal complexes in the organic phase. However, molecules synthesized in experiments often lead to very low efficiency. This suggests that there are high energy barriers between metal complexes in the aqueous and organic phases. It is reasonable to suppose that those energy barriers are located near the interface between the aqueous and organic phases $[1,2]$. In spite of the need for the interface information, it is experimentally difficult to obtain structural details in nanometer order.

The permeation process of the metal complexes through the interface is also related to the energy barrier between the organic and aqueous phases. Because, in general, the permeation process is transient and occurs in a heterogeneous system, it is hard to obtain information of the permeation process by 
experimental measurements in a batch solvent extraction. Thus, the measurement technique with at least $0.1 \mathrm{~ns}$ and $0.1 \mathrm{~nm}$ resolution is needed.

Molecular simulations, such as ab initio molecular dynamics (AIMD) method [8-10], molecular dynamics (MD) method and Monte Carlo (MC) method, are powerful tools for atomistic analysis. However, AIMD requires high computational costs, limiting calculation time and system size. MD is hard to simulate chemical reactions and $\mathrm{MC}$ could not be used to compute dynamics of molecules. Thus, for large systems with chemical reactions, a hybrid MC/MD method has been developed and applied to various fields $[11,12]$. The hybrid MC/MD method is carried out for chemical reaction process by repeating MC and MD calculations, called MC/MD cycles, where MC and MD are used for chemical reactions probabilistically by swapping specific molecules and molecule movements on the short time scale, respectively.

In this paper, permeation process of cobalt(II) (Co) complexes through the interface between aqueous and organic phases was investigated by using the hybrid MC/MD method. All calculations were carried out in heterogeneous biphasic systems composed of organic (cyclohexane (CHX)) and aqueous (water) layers. The hydroxyoxime extractant, LIX63 and the carboxylic acid extractant, Versatic Acid 10 (VA10) were selected as extractant molecules (shown in Figure 1). These extractants are widely used in industry and known to be water insoluble. The rate of Co extraction by LIX63 is faster than that by VA10, and the system with the mixture of LIX63 and VA10 exhibits the synergistic effect [5]. We performed the calculation with three systems (Co-LIX63, Co-VA10 and Co-(LIX63+VA10)) and analyzed these trajectories.<smiles>CCCCC(CC)C(=N[O-])C(O)C(CC)CCCC</smiles>

LIX63<smiles>CC(C)(C)CCCCCC(=O)O</smiles>

VA10

Figure 1. Structures of LIX63 and VA10 used in this study.

\section{Methods}

MD simulations were performed with the GROMACS software package $[13,14]$. The force field parameters, the atomic charges and the calculation conditions used in this study were the same as the previous studies $[15,16]$. The initial model systems were heterogeneous biphasic ones composed with 508 CHX molecules and $\sim 3,300$ water molecules. Twenty-five $\mathrm{Co}^{2+}$ ions, and 55 LIX63 and/or 55 VA10 molecules were embedded randomly in the water and CHX layers, respectively. No other metal ions were added in the systems in order to avoid the competition with the $\mathrm{Co}^{2+}$ extraction and focus on the permeation of the Co complexes through the interface. The systems were embedded in rectangular boxes in a periodic boundary condition, and $\mathrm{H}_{3} \mathrm{O}^{+}$and $\mathrm{Cl}^{-}$were added to neutralize the systems. In addition, $\mathrm{H}_{3} \mathrm{O}^{+}$could stabilize the Co complexes, reported in our previous studies [16]. The box sizes were set to be $4.6 \mathrm{~nm} \times 4.6$ $\mathrm{nm} \times 9.8 \mathrm{~nm}$ and described by the coordinate systems $\mathrm{XYZ}$ with the $\mathrm{Z}$ axis along the interface normal (shown in Figure 2). The pressure of the systems was maintained at 1 bar by Parrinello-Rahman approach 
$[17,18]$, and the temperature was kept at $300 \mathrm{~K}$ and controlled by the velocity-rescaling thermostat [19].

MC molecule swaps were carried out with my own code. In the MC scheme, the transition probability calculated by Metropolis algorithm [20] written as $W_{i \rightarrow s}=\min \left\{1, \exp \left(-\Delta E / k_{\mathrm{B}} T\right)\right\}$ is related to the net energy difference $\Delta E=E_{s}-E_{i}$ of swapped and initial configurations where $k_{\mathrm{B}}$ is the Boltzmann constant and $T$ is temperature.

The hybrid MC/MD method was applied for the long time-scale phenomena, using MD method for short time-scale molecule movements and MC method for long time-scale chemical reactions. The procedure of the hybrid MC/MD method is as follows:

1. molecular modeling and energy minimization,

2. short MD simulation (5 $\mathrm{ns}$ ),

3. selections of swapped molecules and MC swaps,

4. short MD simulation (1 ns), and

5. repeats of 3 and 4 till the pre-defined number (called the MC/MD cycles).

The water molecules within $0.3 \mathrm{~nm}$ around $\mathrm{Co}^{2+}$ ions were swapped with randomly selected LIX63 or VA10 molecules in the organic phase. Every short MD simulation was run on $1 \mathrm{~ns}$ and the molecule swaps occurred when the transition probability $W_{i \rightarrow s}$ was 0.6 or higher. The numbers of the MC/MD cycles in all calculations were set to be 70 .

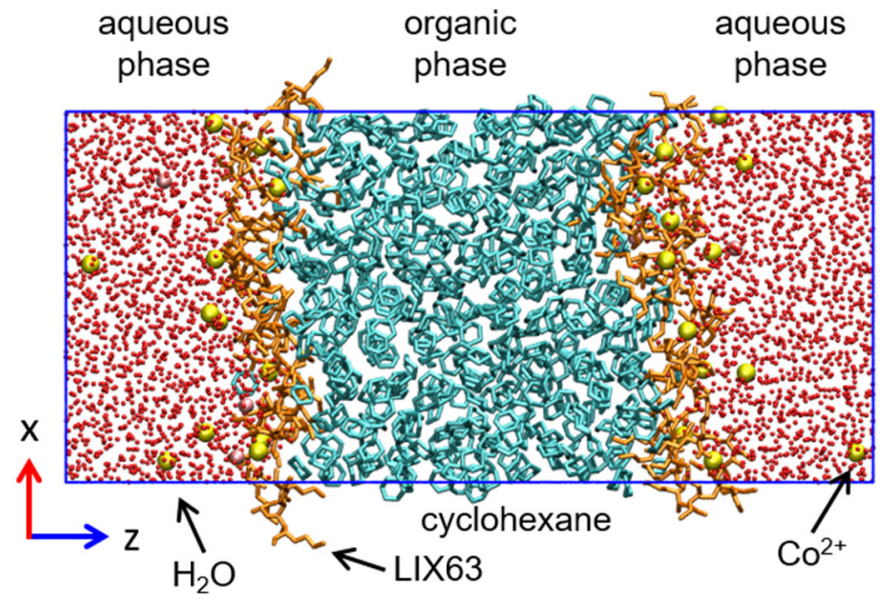

Figure 2. Whole system with $\mathrm{Co}^{2+}$ and LIX63 molecules. $\mathrm{Co}^{2+}$ are represented by the yellow spheres. LIX63, cyclohexane and $\mathrm{H}_{2} \mathrm{O}$ molecules are colored orange, cyan and red, respectively. For simplicity, hydrogen atoms in all molecules are not shown. The interface normal is set to be the $\mathrm{Z}$ axis.

\section{Results and Discussion}

\subsection{Number density distributions of molecules and ions}

Figure 3 shows the number density profiles of the selected components in the systems from $5 \mathrm{~ns}$ short MD trajectories, projected onto the interface normal (Z-axis). In the LIX63 system, LIX63 molecules and $\mathrm{Co}^{2+}$ were located at the interface. However, VA10 molecules were mainly located in the organic phase. In addition, $\mathrm{Co}^{2+}$ were distributed in the whole aqueous phase. The characteristic of these extractants calculated from our MD simulations is good agreement with experimental studies, although LIX63 in our MD simulations was deprotonated. This result suggests that LIX63 is better used for the Co extraction, rather than VA10. 
In the LIX63+VA10 system, LIX63 molecules were located at the interface and VA10 molecules were located in the organic phase. It should be noted that the nitrogen atoms in LIX63 molecules were near the interface, but the oxygen atoms in VA10 molecules were further apart. VA10 molecules might push away water molecules around Co complexes and prevent water molecules from being coordinated to $\mathrm{Co}^{2+}$.

It should be noted that our trajectories also showed the dimers consisting of the extractant molecules. In the systems with LIX63, one LIX63 molecule often formed a weak hydrogen bond to another LIX63 molecule or VA10 molecule in the organic phase or around the interface, but the lifetime of the LIX63-LIX63 or LIX63-VA10 dimers was in sub-nano second order. However, the lifetime of the VA10 dimers in the VA10 system was $10 \mathrm{~ns}$ order because of forming two hydrogen bonds between the acidic hydrogen and the carbonyl oxygen. The stable dimer formation leads that the VA10 molecules were stable in the organic phase.
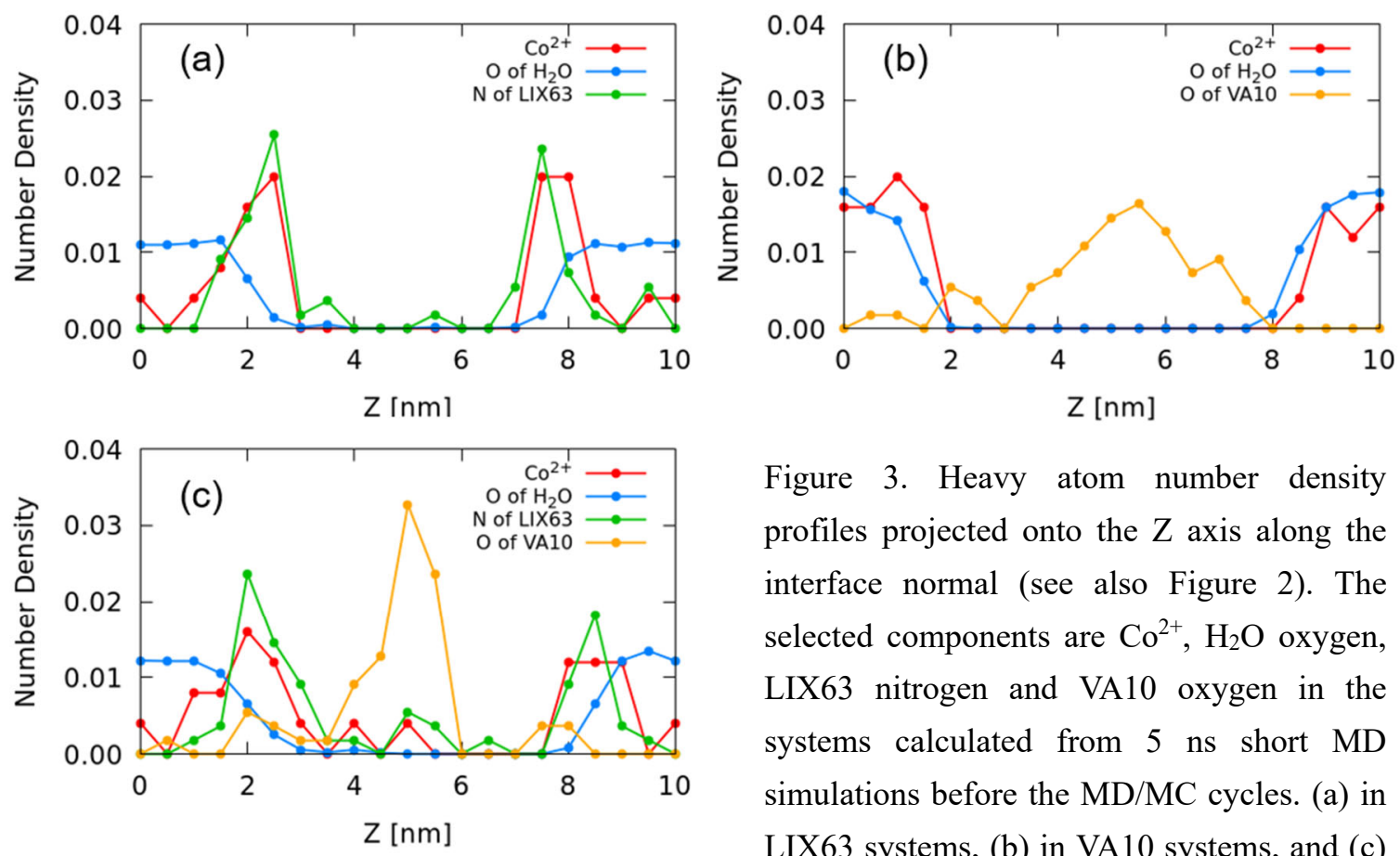

Figure 3. Heavy atom number density profiles projected onto the $\mathrm{Z}$ axis along the interface normal (see also Figure 2). The selected components are $\mathrm{Co}^{2+}, \mathrm{H}_{2} \mathrm{O}$ oxygen, LIX63 nitrogen and VA10 oxygen in the systems calculated from 5 ns short MD simulations before the $\mathrm{MD} / \mathrm{MC}$ cycles. (a) in LIX63 systems, (b) in VA10 systems, and (c) in LIX63+VA10 systems.

\subsection{Permeation processes through the organic-aqueous interface}

Figure 4(a) shows the time evolution of the typical Co positions in the LIX63 system. Though the Co complexes permeated through the interface, they remained around the interface and could not move into the organic phase. Figure 4(b-e) shows the snapshots of the Co complexes permeating through the interface. In the permeation process, two LIX63 molecules were coordinated to $\mathrm{Co}^{2+}$ from the organic phase (shown in Figure 4(c)). The Co complexes rotated and one of LIX63 molecules coordinated to $\mathrm{Co}^{2+}$ was located between $\mathrm{Co}^{2+}$ and the aqueous phase (Figure 4(d)), then the complexes moved to the organic phase a little (Figure 4(e)). The Co complex shown in Figure 4(e) consisted of 2 LIX63 molecules and a water molecule coordinated to $\mathrm{Co}^{2+}$. The Co complexes located in the organic phase (Figure 4(e)), remaining around the 
interface (Figure 4(c) and (b)) and staying in the aqueous phase (Figure 4(b)) account for about 10, 60 and $30 \%$, respectively. The structure shown in Figure 4(e) is good agreement with one of the Co-LIX63 complexes in the organic phase, but not with the most stable structure where the Co complex in the organic phase is composed of 3 LIX63 molecule coordinated to $\mathrm{Co}^{2+}[16]$. Thus, because there were some of $\mathrm{Co}^{2+}$ and LIX63 molecules not permeating through the interface, this complex seems to be in one of local minimal. In order to be in the most stable state, it is necessary for LIX63 molecules to diffuse to the Co complexes and to be exchanged with water molecules coordinated to $\mathrm{Co}^{2+}$ because this exchange increased the hydrophilicity of the Co complexes.
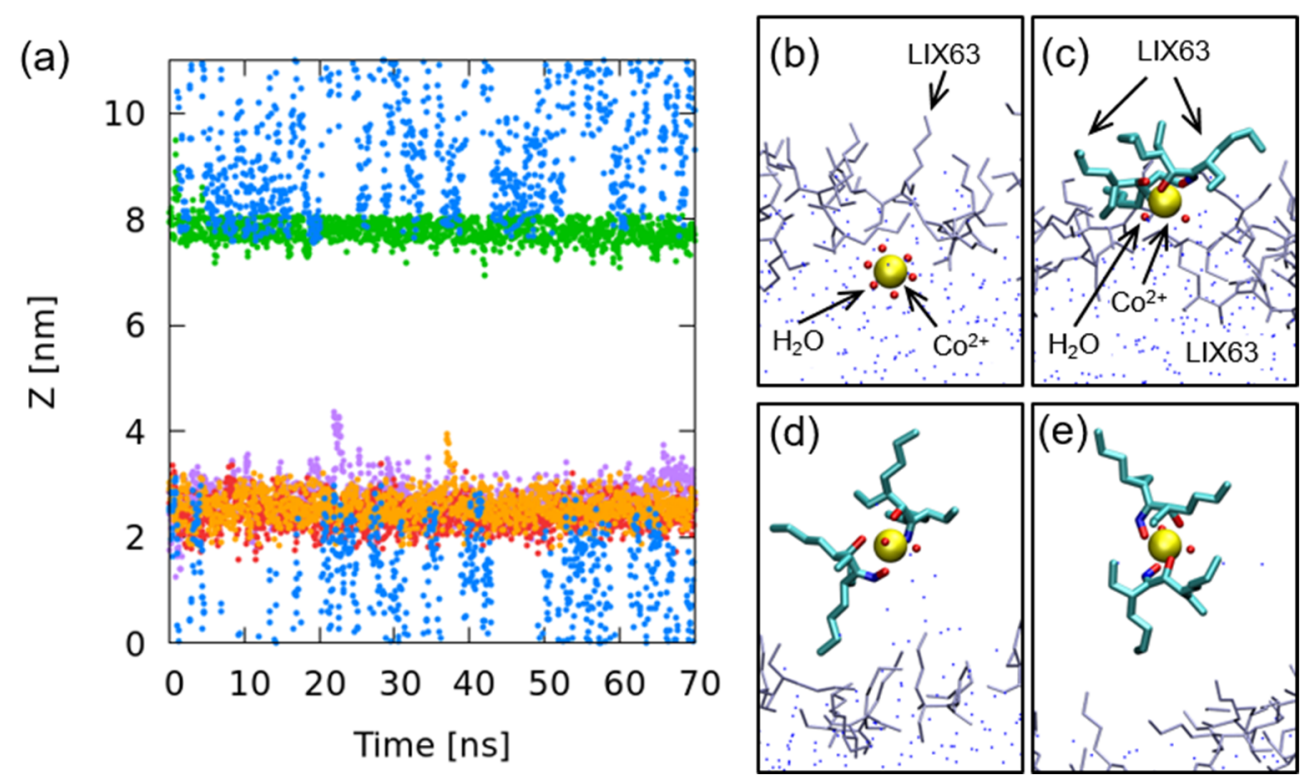

Figure 4. Permeation profiles of Co-LIX63 system. (a) time series of typical 5 Co coordinates along the $\mathrm{Z}$ axis parallel to the interface normal (see also Figure 2), (b-e) snapshots of Co complexes in one trajectory colored with purple in (a). (b) at $0.12 \mathrm{~ns}$, (c) at $21.72 \mathrm{~ns}$, (d) 21.82 and (e) at $21.99 \mathrm{~ns} . \mathrm{Co}^{2+}$ is represented by the yellow sphere. Molecules not coordinated to $\mathrm{Co}^{2+}$ are colored purple (for LIX63) and blue (for $\mathrm{H}_{2} \mathrm{O}$ ). For simplicity, $\mathrm{CHX}$ molecules and hydrogen atoms in all molecules are not shown.

In this study, each LIX63 molecule was set to be a negative charge based on pKa. All LIX63 molecules were located at the interface consequently and no LIX63 molecule could interact with the water molecules coordinated with $\mathrm{Co}^{2+}$ permeating through the interface. Thus, the Co complexes might be unable to move into the organic phase. This result suggests that it is important to know the interface condition in detail experimentally and to apply the condition to the calculation correctively.

$\mathrm{No} \mathrm{Co}^{2+}$ in the Co-VA10 system permeated through the interface (shown in Figure 5(a)). Although water molecules coordinated to $\mathrm{Co}^{2+}$ were exchanged with VA10 molecules in the organic phase by using the hybrid MC/MD method, other water molecules were immediately coordinated to $\mathrm{Co}^{2+}$ and the $\mathrm{Co}$ complexes moved back to the aqueous phase. Our previous study [16] shows that water molecules were mainly coordinated to $\mathrm{Co}^{2+}$ in the Co-VA10 system and VA10 molecules were located at $\sim 0.4 \mathrm{~nm}$ around the Co complexes. Thus, the hybrid MC/MD method might not be suitable for application where extractant 
molecules surrounding metal complexes coordinated with water molecules contribute to the stabilization of the Co complexes.
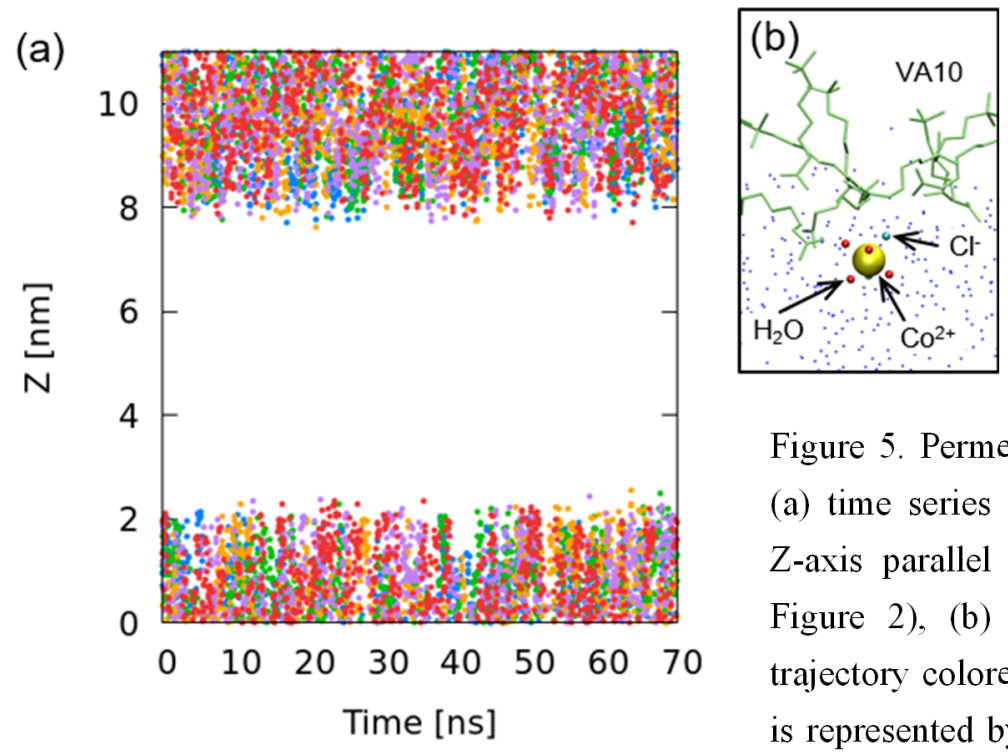

Figure 5. Permeation profiles of Co-VA10 system. (a) time series of typical 5 Co coordinates along Z-axis parallel to the interface normal (see also Figure 2), (b) snapshot of Co complex in one trajectory colored with red in (a) at $32.23 \mathrm{~ns} . \mathrm{Co}^{2+}$ is represented by the yellow sphere. Molecules not coordinated to $\mathrm{Co}^{2+}$ are colored green (for VA10) and blue (for $\mathrm{H}_{2} \mathrm{O}$ ). For simplicity, CHX molecules and hydrogen atoms in all molecules are not shown.

The Co complexes in the Co-(LIX63+VA10) system permeated through the interface and were then located in the organic phase (shown in Figure 6(a)). LIX63 molecules moved to $\mathrm{Co}^{2+}$ from the organic phase and were coordinated to $\mathrm{Co}^{2+}$ at the interface. Then the Co complexes migrated into the organic phase by exchanging coordinated water molecules to LIX63 molecules. Figure 6(b-f) shows the typical complex structures. Two types of the complex structures were obtained, one contained 2 LIX63, 1 VA10 and 1 water molecules (Figure 6(e)), and another contained 2 LIX63 and 1 VA10 molecules (Figure 6(f)). We found hydrogen bonds of these coordinated molecules with extractant and water molecules which were not coordinated with $\mathrm{Co}^{2+}$ (shown as in Figure 6(e)). These molecules consisting of the hydrogen bonds were located at $\sim 0.5 \mathrm{~nm}$ around $\mathrm{Co}^{2+}$, contributing to the stabilization of the Co complexes. However, VA10 molecules were also found at the region and this is not agreement with our previous study [16]. Thus, the Co complexes in this calculation are transient and this result suggests that longer calculation time should be needed for the Co complexes in equilibrium.

Though it is important to know the interface conditions because the interface condition affects the structures of the complexes, it is difficult to obtain information on the interface experimentally. In all calculations, parameters experimentally and theoretically based on bulk information were used. Thus, the observations in the calculations (i.e., LIX63 molecules extensively located at the interface) might be caused by an incorrect representation of the interface condition. It has been reported experimentally that water molecules are ionized in gas-liquid interface, but it is still unclear that the interface is with positive (mainly $\mathrm{H}_{3} \mathrm{O}^{+}$) or negative (mainly $\mathrm{OH}^{-}$) charge because of depending on the compounds in the aqueous solution. 
Thus, more experimental information on the interface is necessary for the investigation of the permeation processes.
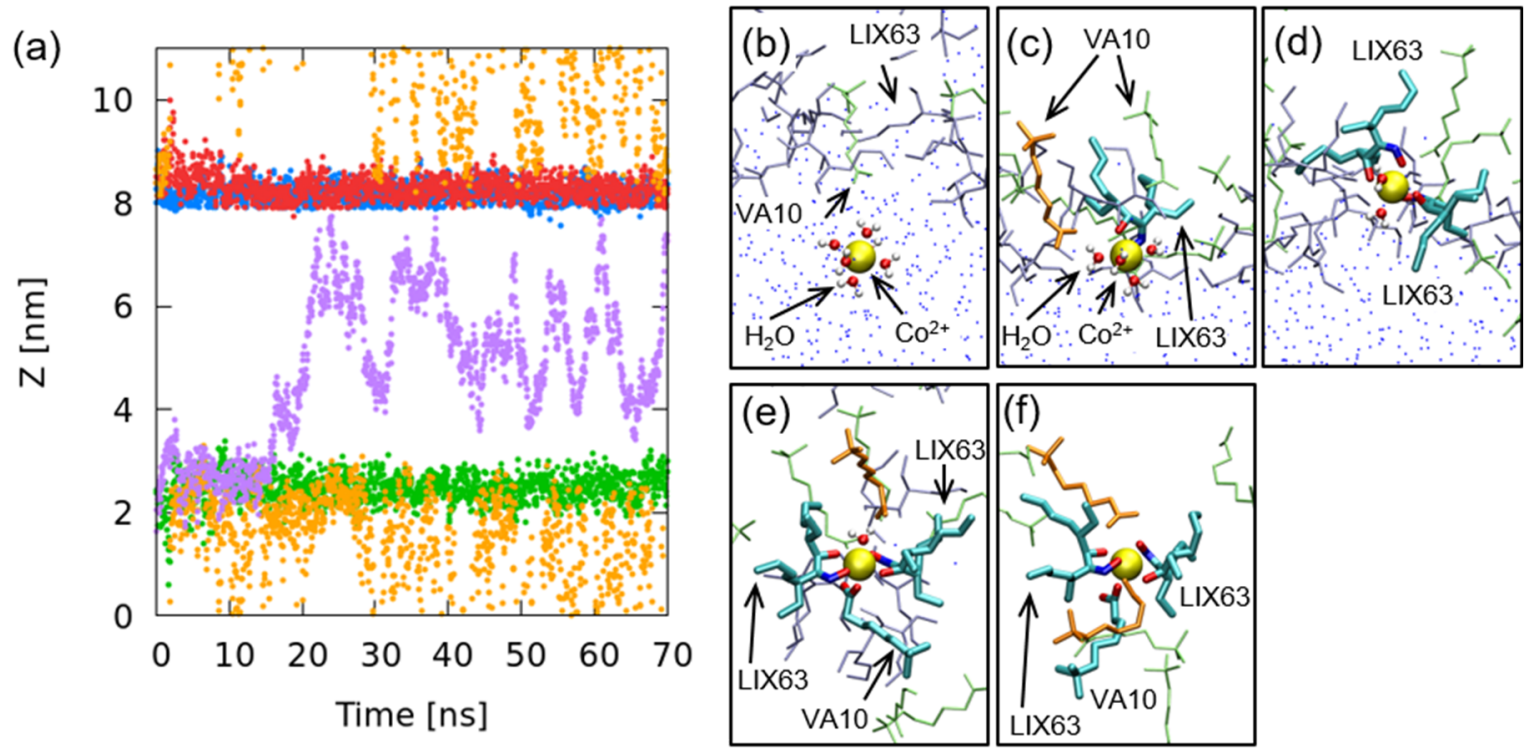

Figure 6. Permeation profiles of Co-(LIX63+VA10) system. (a) time series of typical 5 Co coordinates along Z-axis parallel to the interface normal (see also Figure 2), (b-f) snapshots of Co complex in one trajectory colored with purple in (a), (b) at $2.63 \mathrm{~ns}$, (c) at $8.26 \mathrm{ns,} \mathrm{(d)} \mathrm{at} 9.00 \mathrm{~ns}$, (e) at $11.44 \mathrm{~ns}$ and (f) at $16.92 \mathrm{~ns} . \mathrm{Co}^{2+}$ is represented by the yellow sphere. Molecules not coordinated to $\mathrm{Co}^{2+}$ are colored purple (for LIX63), green (for VA10) and blue (for $\mathrm{H}_{2} \mathrm{O}$ ). VA10 molecules colored orange are not coordinated to $\mathrm{Co}^{2+}$, but located at $\sim 0.5 \mathrm{~nm}$ around $\mathrm{Co}^{2+}$. For simplicity, CHX molecules and hydrogen atoms in all molecules are not shown, except for $\mathrm{H}_{2} \mathrm{O}$ molecules coordinated to $\mathrm{Co}^{2+}$.

In the development of novel extractant molecules, chemical formulas of candidate molecules are determined based on structural and energetic estimation of the metal complexes coordinated with the molecules [7]. However, in many cases, extraction efficiencies measured with synthesized molecules are much lower than ones predicted. This suggests that it is possible not only that the predicted structures of the complexes in the organic phase are incorrect, but also that almost of the complexes could not permeate through the interface. Thus, for the development of novel extractant molecules, it is important to consider the diffusion of extractant molecules on the interface like LIX63 and VA10 molecules, in addition to the coordination of extractant molecules to metal ions like LIX63 molecules. For the examination of small molecule permeation across lipid membrane, free energy profiles along the membrane normal are useful [21]. For the solvent extraction, the height of the free energy barriers between the organic and aqueous phases are related to the extraction rate. The free energy profiles of $\mathrm{Co}^{2+}$ permeating through the organic-aqueous interface are being calculated and will be reported elsewhere. 


\section{Conclusion}

We investigated the permeation process of the Co(II) complexes with LIX63 and/or VA10 extractants through the organic-aqueous interface using the hybrid MC/MD method. The results show that for the LIX63 system, LIX 63 molecules were coordinated to $\mathrm{Co}^{2+}$ from the organic phase, and the Co complexes, which still included water molecules, rotated at the interface, then moved into the organic phase. For the LIX63+VA10 system, it was found that, in addition that LIX63 molecules were coordinated to $\mathrm{Co}^{2+}$ from the organic phase, VA10 prevented water molecules not coordinated to $\mathrm{Co}^{2+}$ from having access to $\mathrm{Co}^{2+}$. The usage of multiple extractants playing the different roles might accelerate the permeation of the Co complexes through the interface.

In the development of novel extractant molecules, the extractant efficiency using new synthesized molecules as extractant candidates is often much lower than that predicted. The results calculated with the hybrid MC/MD method suggest that, in the molecule design for novel extractants, it is necessary to consider structures and stabilization of the metal complexes not only in the organic phase, but also in the permeation through the organic-aqueous interface.

\section{Acknowledgement}

We thank Dr. Satoshi Asano and Mr. Masatoshi Takano at Sumitomo Metal Mining, Co., Ltd. for their discussion.

The molecular images in this article were created by VMD [22].

\section{References}

1) W. J. Albery, J. F. Burke, E. B. Leffler, J. Hadgraft, J. Chem. Soc., Faraday Trans. 1, 72, 1618-1624 (1976).

2) J. P. Simonim, L. Perrigaud, K. Perrigaud, T. H. Vu, Solvent Extr. Ion Exch., 32, 365-377 (2014).

3) M. Duvail, S. Damme, P. Guilbaud, Y. Chen, T. Zemb, J. Dufreche, Soft Matter, 13, 5518-5526 (2017).

4) B. Qiao, J. V. Muntean, M. O. de la Cruz, R. J. Ellis, Langmuir, 33, 6135-6142 (2017).

5) C. Y. Cheng, Hydrometallurgy, 84, 109-117 (2006).

6) M. Takano, Y. Ozaki, S. Asano, Y. Baba, F. Kubota, M. Goto, J. MMIJ, 12, 567-571 (2015).

7) K. Shimojo, I. Fujiwara, K. Fujiwara, H. Okamura, T. Sugita, T. Oshima, Y. Baba, H. Naganawa, Solvent Extr. Res. Dev., Jpn., 23, 151-159 (2016).

8) R. Car, M. Parinello, Phys. Rev. Lett., 55, 2471-2474 (1985).

9) M. E. Tuckerman, J. Phys.: Condens. Matter, 14, R1297-R1355 (2002).

10) M. C. Payne, M. P. Teter, D. C. Allen, T. A. Arias, J. D. Joannopoulos, Rev. Mod. Phys., 64, 1045-1097 (1992).

11) M. Widom, W. P. Huhn, S. Maiti, W. Seurer, Metall. Mater. Trans. A, 45, 196-200 (2014).

12) M. Nagaoka, Y. Suzuki, T. Okamoto, N. Takenaka, Chem. Phys. Lett., 583, 80-86 (2013).

13) H. J. C. Berendsen, D. van der Spoel, R. van Drunen, Comput. Phys. Commun., 91, 43-56 (1995).

14) M. J. Abraham, T. Murtola, R. Schulz, S. Pa'll, J. C. Smith, B. Hess, E. Lindahl, SoftwareX, 1-2, 19-25 (2015).

15) Y. Nishihara, S. Yoshio, K. Maki, Solvent Extr. Res. Dev., Jpn., 25, 59-69 (2018). 
16) Y. Nishihara, S. Yoshio, Solvent Extr. Res. Dev., Jpn., 27, 39-47 (2020).

17) M. Parrinello, A. Rahman, J. Appl. Phys., 52, $7182-7190$ (1981).

18) S. Nosé, M. L. Klein, Mol. Phys., 50, 1055-1076 (1983).

19) G. Bussi, D. Donadio, M. Parrinello, J. Chem. Phys., 126, 014101 (2007).

20) N. Metropolis, A. W. Rosenbluth, M. N. Rosenbluth, A. H. Teller, E. Teller, J. Chem. Phys., 21 1087-1092 (1953).

21) B. L. de Grot, H. Grubmuller, Science, 294, 2353-2357 (2001).

22) W. A. Humphrey, A. Dalke, K. Schulten., J. Mol. Graphics, 14, $27-28$ (1996). 